\title{
De la crisis de la energía al concepto del patrimonio económico de la humanidad: criterios para reestructurar el sistema sconómico internacional *
}

Las sucesivas crisis que han venido estremeciendo el sistema económico internacional en los últimos años, revelan palpablemente su inadecuación frente a los requerimientos de la sociedad internacional contemporánea, en su compleja dimensión política, social, cultural y propiamente económica. Si solamente se tiene presente el flujo y reflujo de la crisis monetaria, las dislocaciones que ella ha introducido en el comercio internacional y, sobre todo, la crisis de la energía, se podrá apreciar la magnitud que adquiere este fenómeno. Más significativa todavía es, en este sentido, la inestable relación entre los diferentes bloques económicos y las muy insatisfactorias respuestas que han recibido los problemas de los países en desarrollo, todo lo cual amenaza con proyectar las actuales crisis parciales, sectoriales o transitorias, hacia una alteración generalizada de impredecibles consecuencias.

La reacción normal frente a las crisis aludidas ha sido la de buscar respuestas parciales, frecuentemente mirando exclusivamente al sector donde se manifiesta la crisis y muchas veces sobre bases ad hoc o inclusive temporales. Para mencionar sólo algunos ejemplos de esta situación, puede señalarse el caso de la creación de los derechos especiales de giro, que miraba fundamentalmente a solucionar problemas de liquidez y presiones sobre las balanzas de pago $^{1}$; las iniciativas de reforma del sistema monetario internacional, cuya vinculación con la reforma del sistema de comercio internacional no aparece del todo clara $^{2}$; la reforma del GATT, aparentemente con la intención de

- Estudio presentado a la Reunión Regional sobre empresas multinacionales, organizada por The American Society of International Law y The Law School, University of Virginia, Charlottesville, Va., marzo de 1974.

"Para los antecedentes que condujeron a la creación de los derechos especiales de giro y sus objetivos, Joseph Gold: Special Drawing Rights, International Monetary Fund, Pamphlet Series, No 13, 1969.

"Sohre la necesidad de una estrecha relación entre las negociaciones monetarias y comerciales, Guy F. Erb: "New Trade and Monetary Systems", Overseas Development Council: The Uniled States and the developing world. Agenda for Action, 
mejorar todavía más la posición de predominancia de los países desarrollados ${ }^{3}$; o la creación de un sistema de preferencias en la creencia que se trata de la solución final para los problemas y necesidades de los países en desarrollo. ${ }^{4}$

Pero por más inteligentes que sean las respuestas diseñadas, el solo hecho de estar ellas concebidas en un contexto parcial determina su imposibilidad de atacar los problemas de fondo subyacentes a todo el sistema económico internacional. Cada crisis parcial o sectorial tiene su origen en una causa común, que no es otra que el hecho que las premisas económicas fundamentales en que descansó el sistema de los acuerdos de Bretton Woods, y antes de ellos el esquema económico del siglo $\mathrm{xIx}$, y sus correspondientes principios e instituciones jurídicas, han perdido su razón de ser y su operatividad, si acaso no su legitimidad, en una sociedad internacional radicalmente diferentes de aquella que los concibió. De ahí que cualquier solución que aspire a ser permanente debe necesariamente ser global $y$, por tanto, tomar en cuenta la realidad que significa la presencia de los países en desarrollo, que precisamente han sido el factor fundamental del cambio operado en la sociedad internacional.

El presente estudio tiene por objeto, por una parte, examinar las causas que han llevado a la obsolecencia de las premisas económicas y los principios jurídicos del sistema actualmente imperante $y$, por otra parte, explorar las bases y tendencias en que eventualmente pueda descansar la reestructuración general de la economía internacional, en función de la realidad contemporánea.

Washington, D.C., 1973, pp. 31-34. Los paises en desarrollo han insistido constantemente en esta relación. Véase por ejemplo, la Evaluación de Quito sobre la Estrategia Internacional de Desarrollo, especialmente sección $\mathbf{H}$, aprobada por la Resolución 320 (XV) de CEPAL, del 29 de marzo de 1973.

${ }^{8}$ Los países en desarrollo han hecho presente que las nuevas negociaciones comerciales multilatcrales de 1973 no buscan satisfacer adecuadamente sus problemas. Véase, por ejemplo, la Resolución 4 (XV) de la Comisión Especial de Coordinación Latinoamericana (CECLA), de enero de 1973, sobre "Principios de Política para la fase preparatoria de las negociaciones comerciales multilaterales de 1978", especialmente sus considerandos.

* Si bien los sistemas preferenciales de la CEE y Japón, y aquel que cventualmente establezca Estados Unidos, constituyen un paso positivo respecto de las exportaciones de productos manufacturados y semimanufacturados de los países en desarrollo, se requieren además otras medidas adicionales para su cficiente aprovechamiento. Véase en este sentido, Erb, loc. cit., Nota 2 supra, pp. 35-37. 


\section{1. - LAS GONSEGUENGIAS DEL LIBRE GAMBISMO}

El postulado central en que descansó la estructura económica tradicional, según ella llegó a consagrarse en el siglo xix, fue la libertad del mercado y la división internacional del trabajo, materializadas por medio de la cláusula de la nación más favorecida como su herramienta ${ }^{5}$. Teóricamente todos los países derivarían iguales o similares ventajas de este sistema, sobre la base de su eficiencia relativa y de la libre competencia, igualdad de acceso a los mercados y no discriminación. Sin embargo, la teoría sería fuertemente contradicha por la práctica.

El sistema clásico prescindió por completo del hecho de que el diferente grado de desarrollo determinaba una diferente capacidad de aprovechamiento del mercado internacional. Mientras para los países poderosos en lo industrial, político y militar, ese mercado ofrecía todas las ventajas, comenzando por la libertad de acceso a las materias primas y culminando en la amplia colocación de productos elaborados, para aquellos otros países de escasa o ninguna industrialización el sistema se reducía a una corriente de intercambio cuyos términos serían cada vez menos favorables y, por tanto, de poca significación como estímulo a su propio proceso de desarrollo interno. De esta manera llegó a consolidarse el conocido fenómeno de los países en desarrollo como proveedores de materias primas e importadores de productos industrializados ${ }^{6}$, en un tipo de relación formalmente distinto pero sustantivamente idéntico al que había existido en los períodos de dominación colonial entre las metrópolis y sus territorios de ultramar.

Diversos factores agravantes también incidieron en este cuadro. Uno de ellos fue la creencia de que las materias primas, así como

'Para un análisis del funcionamiento de la cláusula de la nación más favorecida en el sistema tradicional, con particular referencia a la experiencia de América Latina, Francisco Orrego Vicuña (ed.): América Latina y la cláusula de la nación más favorecida, Dotación Carnegie para la Paz Internacional, Santiago de Chile, 1972.

'Para los países desarrollados de economfa de mercado, los productos primarios representaron en 1969 un $23,2 \%$ dc sus exportacioncs totales, en tanto que los productos manufacturados representaron un 76,9\%. Para los países en desarollo estas cifras son las inversas: los productos primarios representaron un $76,2 \%$ de sus exportaciones y los manufacturados un 23,8\%. Para los países socialistas estas cifras fueron respectivamente el $35,7 \%$ y el $64,3 \%$. Ovcrseas Development Council, op. cit., Nota 2 supra, p. 127. 
los recursos del mar, eran inagotables, lo que contribuyó a asignarles un valor pequeño y reducir en consecuencia sus precios y condiciones de compra a un mínimo. Otro de estos factores fue el hecho de que los propios paises afectados rara vez tomaron conciencia clara de las consecuencias que derivaban del sistema $y$, por tanto, poco o nada pudieron influir en la introducción de mecanismos correctivos; un ejemplo preclaro en este sentido fue el de la cláusula Bello, por medio de la cual se procuró establecer un sistema de preferencias regionales entre los países latinoamericanos cuyos beneficios no se harían extensivos a terceros, iniciativa que no prosperó ${ }^{7}$. En cambio, los países industrializados percibieron claramente las ventajas y desventajas involucradas, como lo prueba el hecho de que formaron su base industrial sobre la base de la protección de su economía nacional y, sólo cuando ésta estuvo consolidada, adhirieron a las reglas del libre cambio para proyectarse en el ámbito internacional ${ }^{8}$; esta percepción facilitó de sobremanera su habilidad para dirigir e influir el funcionamiento del mercado, asegurando bases estables para su prosperidad.

A modo de resumen, cabe indicar que el funcionamiento del sistema económico librecambista produjo resultados diametralmente diferentes para los países industrializados y para los países en desarrollo. Para los primeros aseguró el libre acceso a las materias primas, ya sea mediante su propia política de inversiones extranjeras o a través de la simple compra de los únicos bienes que los países en desarrollo estaban en condiciones de producir, todo ello a precios mínimos; paralelamente aseguró la colocación no discriminada de su producción industrial en el mercado internacional, bajo el marco de la protección de la cláusula de la nación más favorecida y, cuando necesario, por la fuerza de las armas. En cambio, para los países en desarrollo significó la imposibilidad de crear una base industrial propia eficiente $\mathrm{y}$, más aún, tampoco aseguró su acceso no discriminado a los mercados de los países industrializados, pues estos últimos en la práctica lograron establecer procedimientos y mecanismos discriminatorios, inclusive respecto de aquellos productos primarios que no eran de su interés.

Consecuencia de todo ello fue una enorme concentración de rique-

${ }^{7}$ Véase Francisco Orrego Vicuna: "Estudio sobre la cláusula Bello y la crisis de la solidaridad latinoamericana en el siglo xIX". En op. cit., Nota 5 supra, pp. 33-95.

${ }^{8}$ Como un ejemplo de ello, cabe señalar que Estados Unidos sólo adhirió al sistema incondicional de la cláusula de la nación más favorecida en 1923. Véanse las instrucciones del Secretario de Estado Hughes al cuerpo diplomático norteamericano, del 18 de agosto de 1923. En Bishop: International Law. Cases and Materials, 1962, pp. 152-153. 
zas y poderío económico en los países industrializados, y el nacimiento y progresión de la brecha que cada día los ha distanciado más de los países en desarrollo ${ }^{9}$. Este resultado es inevitable cuando una parte poderosa y una parte débil entran en un esquema de libre competencia o, en otras palabras, cuando partes económicamente desiguales miden sus derechos y obligaciones en función de normas jurídicamente iguales. Por esta razón es que cuando un Estado fundamenta su sistema económico nacional en un esquema de libre competencia, lo hace siempre acompañado de mecanismos correctivos destinados a precaver los efectos adversos que puedan generarse para los grupos de bajos ingresos dentro de esa sociedad; entre estos mecanismos cabe mencionar, a título de ejemplo, la legislación social que protege al débil del poderoso -que es un caso de aplicación de normas jurídicas desiguales a partes económicamente también desiguales-, la prohibición de prácticas restrictivas o discriminatorias y, sobre todo, la redistribución del ingreso en forma progresiva para evitar la concentración de la riqueza y satisfacer una elemental justicia social, que a la vez es el requisito indispensable de la convivencia. Sin embargo, ninguno de estos mecanismos correctivos existe en el caso del sistema económico internacional, lo que, como se verá, ha sido uno de los elementos inductivos de su crisis actual.

\section{2. - BRETTON WOODS: LOS CAMBIOS FORMALES}

Los acuerdos de Bretton Woods simbolizaron una primera tentativa de reestructurar el sistema económico internacional. Sin embargo, la

${ }^{\circ}$ Las siguientes cifras son claramente demostrativas de esta brecha:

Paises en desarrollo

Producto bruto per cápita

Poblacion (millones)

Alfabetismo

Consumo de proteinas

(gramos por persona)

Consumo de calorías

Expectativa de vida

Mortalidad infantil

(por mil nacimientos con vida)

Consumo de electricidad

per cápita (KWH al año)

$$
\begin{gathered}
\$ 230 \\
1.850 \\
40 \% \\
54 / \text { día } \\
2.180 / \text { dia } \\
52 \text { años }
\end{gathered}
$$

110

220

\section{Paises desarrollados}

$\$ 3.085$

664

$97 \%$

$97 /$ día

$3.030 /$ dia

71 años

Adaptado de Overseas Development Council, op. cit., Nota 2 supra, p. 123. 
preocupación central que ellos tuvieron fue la de restablecer el funcionamiento de la economía internacional luego de haber sido gravemente dañada por los efectos de la segunda guerra mundial; de ahi que el énfasis principal fuera el fomento y la reconstrucción, particularmente de los países afectados por la guerra, que representaban la infraestructura industrial de la sociedad internacional. Paralelamente, el objetivo expresamente declarado de estos acuerdos y de otros esfuerzos fue la preservación de la paz ${ }^{10}$, lo que tácitamente suponía concentrar la acción en los países capaces de hacer la guerra, esto es, nuevamente la perspectiva se inspiraba en las necesidades del mundo industrializado.

Lo anterior explica porque Ios acuerdos de Bretton Woods en ningún momento cuestionaron las bases mismas en que descansaba el sistema económico internacional, sino que retomando los principios esenciales que venían proyectándose desde el siglo xIx, introdujeron variantes sólo en su manera de operar, en el aspecto técnicaformal, pero no en la esencia, que continuó siendo la misma. La principal modificación respecto del esquema que prevalecía con anterioridad a la guerra, fue la creación de mecanismos e instituciones multilaterales, en contraposición a la técnica principalmente bilateral del periodo anterior ${ }^{11}$; la técnica multilateral se hacía necesaria tanto por el mayor número de Estados y de situaciones que se hacían presentes en un medio más complejo, como por la concepción de que una paz duradera exigía de la participación de todos los Estados en ese sistema. ${ }^{12}$

Pero, si además de lo formal, se examina el significado de los nuevos mecanismos, se podrá observar que ellos mantienen la misma situación anterior. En primer lugar, todos ellos consagran la preponclerancia de los países industrializados en la conducción del sistema, tanto al nivel político - como en el Consejo de Seguridad de las $\mathrm{Na}$ ciones Unidas-, como en el nivel propiamente económico; el mecanismo de clecisión en el Fondo Monetario o en el Banco Internacional, entre otros casos, son un ejemplo de esto último ${ }^{13}$. En segundo lugar,

${ }^{10}$ John H. Jackson: World Trade and the Law of GATT, 1969, pp. 37-39.

${ }^{11}$ Un ejemplo característico del bilateralismo fueron los Reciprocal Trade Agrcements de los Estados Unidos. Según observa Jackson, prácticamente todas las cláusulas del GATT encuentran su origen en ellos. Ibid., p. 37; ello es una demostración de que la naturaleza del cambio fue meramente formal.

${ }^{12}$ Además, las negociaciones multilaterales precavían que terceros países, beneficiarios de la cláusula de la nación más favorecida, pudieran gozar gratuitamente de las concesiones otorgadas en los convenios bilaterales. Jackson, op. cit., p. 40. De esta manera, ninguna concesión hecha por un país desarrollado dejaría de tener una contrapartida.

${ }^{13}$ Ello no solamente se traduce en una concentración del poder decisorio en los países desarrollados, sino que además se traduce en consecuencias económicas 
el acceso no discriminado de los productos industriales al mercado mundial se ve reafirmado a través de la garantía multilateral de la cláusula de la nación más favorecida en el GATT y otras negociaciones tarifarias, fenómeno que llega a abarcar a los países socialistas en el marco de la détente que sobrevino a la guerra fría. $Y$ en tercer lugar, el acceso a las materias primas continúa inalterado ${ }^{14}$, registrándose importantes corrientes de inversión en este plano. ${ }^{15}$

La situación de los países en desarrollo dentro del sistema continúa también su deterioro progresivo. Los precios y condiciones de las materias primas continúan su descenso, frente al aumento de los precios de los productos industriales ${ }^{16}$. El acceso de la producción de los países en desarrollo al mercado mundial continúa sujeto a discriminación, no solamente desde el punto de vista de las barreras no arancelarias ${ }^{17}$, sino también desde el punto de vista de la multilateralidad del GATT, puesto que siempre los países en desarrollo han tenido una participación marginal debido a que no producen ni consumen los principales productos objeto de las negociaciones tarifarias, ni tampoco cuentan con un poder de represalia efectivo para precaver la aplicación de medidas discriminatorias ${ }^{18}$. Incluso cabe observar que el único mecanismo del sistema de postguerra que en alguna medida se preocupó de la situación de los países en desarrollo, cual fue la Carta de La Habana y la proyectada Organización

concretas: un ejemplo es el hecho de que los países en desarrollo sólo han obtenido un $25 \%$ del total de los derechos de giro asignados a los miembros del Fondo Monetario, proporción determinada por sus cuotas en este organismo.

1* Al respecto debe tenerse presente que uno de los objetivos centrales de la Carta del Atlántico, de agosto de 1941, que dio inicio al sistema de postguerra, fue el de "...incrementar el goce por todos los Estados, grandes o pequeños, vencedor o vencido, en el acceso, en iguales términos, al comercio de las materias primas del mundo que son necesarias para la prosperidad económica". Jackson, op. cit., p. 38.

${ }^{15} \mathrm{Las}$ inversiones extranjeras directas de los Estados Unidos en actividades extractivas aumentaron de 5.020 millones de dólares valor de libros en 1951 a 15.212 millones en 1961; en 1971 alcanzaron a 30.978 millones. Ello equivale a un aumento porcentual del $203 \%$ en el período $1951-1961$ y del $104 \%$ en el perfodo 1961-1971. Véase Lincoln Gordon: "Multinational Corporations and the Less Developed Countries", Düsseldorf Conference on the International Control of Investment, enero 1973, p. 8.

${ }^{10}$ Sobre los problemas que afectan al comercio de los países en desarrollo, véase en general Harry H. Johnson: Economic Policies Toward Less Developed Countries, Praeger, 1967.

${ }^{13}$ Ibid., pp. 78-110.

${ }^{18}$ Celso Lafer: "El GATT, la cláusula de la nación más favorecida y América Latina". En op. cit,, Nota 5 supra, pp. 123-148. Este estudio demuestra igualmente que la incorporación de la Parte IV del GATT no ha tenido en la práctica influencia alguna en la siluación de los paises en desarrollo. 
Internacional de Comercio, fue precisamente el que nunca llegó a materializarse. ${ }^{19}$

Tampoco el esquema de libre competencia fue objeto de correcciones destinadas a precaver sus efectos adversos para los países en desarrollo y, menos aún, acompañado de algún grado de redistribución del ingreso en el plano internacional. El énfasis puesto durante este periodo en la ayuda internacional corresponde más bien a una concepción caritativa que a una política real de estímulo para el desarrollo, aparte de los problemas derivados de su uso político y, en cualquier caso, de su absoluta insuficiencia. ${ }^{20}$

Un sistema económico de esta naturaleza llevaba en sí el germen de su propia crisis, principalmente por el hecho de no haber tomado en cuenta la realidad que ya era visible en la postguerra, en que un número creciente de países iniciaba un proceso de presión destinado a asegurar su participación equitativa en el medio internacional. Esta tendencia sería la que gradualmente configuraría el fenómeno del tercer mundo. Por otra parte, el fomento masivo de la inversión privada extranjera daría lugar a otro fenómeno que, después de algunos años, repercutiria en la propia estabilidad del sistema de Bretton Woods: las empresas multinacionales. Pero antes de examinar estos aspectos, interesa referirse a algunos esfuerzos que han procurado estructurar sectores determinados del sistema económico sobre bases diferentes a las tradicionales.

\section{3. - LA BÚSQUEDA DE PRINCIPIOS ALTERNATIVOS}

En el curso del siglo $x \mathbf{x}$ se han visto surgir diversos mecanismos que, de una u otra manera, han procurado estructurar sectores determinados sobre la base de principios diferentes a aquellos que inspira-

${ }^{10} \mathrm{La}$ Carta de La Habana contenfa capitulos referentes a "Empleo y Actividad Económica", "Desarrollo Económico y Reconstrucción", "Prácticas de Negociaciones Restrictivas" y "Acuerdos Intergubernamentales de Productos Primarios", entre otras materias que el GATT no contempló. Propuestas de Chile, Cuba, Brasil e India estuvieron dirigidas a incluir un capítulo sobre carteles y sobre reglamentación de los servicios; igualmente Brasil e India abogaron por un estricto control de las restricciones cuantitativas; diversas otras proposiciones procuraron atender los problemas de los países en desarrollo. Véase Celso Lafer, loc. cit., Nota 18 supra, pp. 124-130.

${ }^{20}$ El flujo neto de asistencia oficial para el desarrollo, como porcentaje del producto nacional bruto, ha disminuido progresivamente: en 1961 alcanzaba a un $0,53 \%$; en 1971 sólo a un 0,35\%. Overseas Development Council, op. cit., Nota 2 supra, p. 159. 
ban el sistema económico tradicional. Algunos de estos mecanismos han significado apartarse de las reglas de la libre competencia, en tanto que otros han introducido el tratamiento diferencial en función de los distintos grados de desarrollo económico de las partes involucradas.

\section{El Comercio de Estado}

Cronologicamente, el primero de estos mecanismos ha sido el del comercio de Estado. En rigor no se trata éste de un principio que haya regulado las relaciones económicas al nivel internacional sino que una forma de organización de la economía nacional sobre la base del monopolio estatal, general o parcial. Sin embargo, al cerrar un determinado mercado al libre juego de la competencia, necesariamente ha repercutido en la forma como se desarrollan las relaciones económicas internacionales, principalmente desde el punto de vista de la inaplicabilidad de la cláusula de la nación más favorecida en el seno de ese mercado. De ahí que, por regla general, los países que practican el comercio de Estado hayan organizado sus transacciones internacionales en función de un "quid pro quo", esto es, intercambiando productos por valores monetarios equivalentes, lo que muchas veces ha tomado la forma de trueque. Consecuencia de ello, por lo menos hasta hace poco tiempo, ha sido también la no participación de estos países en los mecanismos multilaterales de la postguerra.

Desde el punto de vista del interés de los paises en desarrollo, el comercio de Estado no constituye como tal principio una respuesta a sus necesidades. El hecho de que la relación descanse en un "quid pro quo", que significa un tratamiento idéntico para las partes, impide a los países en desarrollo obtener el trato preferencial requerido por su menor grado de desarrollo económico, con lo cual continúan sujetos a la igualdad jurídica formal en términos similares a lo que ocurre en el sistema de la cláusula de la nación más favorecida ${ }^{21}$. Por otra parte, tampoco el comercio de Estado garantiza el acceso no discriminado de los productos de los países en desarrollo a los mercados estatizados, por cuanto en esencia se trata de una herramienta discriminatoria ${ }^{22}$. Además, las operaciones de las empresas estatales en el

${ }^{21}$ Francisco Orrego Vicuña: "El comercio de Estado: alternativa de la cláusula de la nación más favorecida en la estructuración del comercio internacional". En Francisco Orrego Vicuña (ed.): Ensayos sobre derecho internacional econdmico, Vol. II, Mexico, Fondo de Cultura Económica (En prensa).

${ }^{22}$ En 1970 las exportaciones de los países en desarrollo a los palses socialistas alcanzaron sólo a un $5,8 \%$ de sus exportaciones totales. Overseas Development Council, op. cit., Nota 2 supra, p. 130. 
mercado internacional en poco o nada se diferencian de las operaciones de las empresas privadas y multinacionales, con lo cual la posibilidad de prácticas restrictivas continúa igualmente presente. ${ }^{23}$

Por las razones anteriores, el comercio de Estado no constituye una alternativa que pueda superar los problemas de fondo del sistema económico internacional, aun cuando en determinados casos pueda contribuir a mejorar la posición relativa de los países en desarrollo que lo practican, por ejemplo mediante el uso más eficiente de su poder de compra. ${ }^{24}$

Acuerdos sobre productos básicos

Otro mecanismo que interesa destacar es el de los acuerdos sobre productos básicos, los cuales en cierta medida tienden a superar el principio de la libre competencia y de la oferta y la demanda, para fundamentarse en otras bases. En efecto, la primera característica de estos acuerdos es que descansan en un grado de institucionalización, aun cuando elemental. Sus instituciones reúnen tanto a los países productores como a los países consumidores de un producto determinado, procurante conciliar sus respectivos intereses mediante una cierta planificación del sector, particularmente en lo que se refiere a los volúmenes de producción y a los precios de compra. ${ }^{25}$

$\mathrm{Si}$ bien en teoría estos acuerdos garantizarían tanto el interés de los países productores en desarrollo, que obtendrían precios estables, como el interés de los países consumidores desarrollados, que obtendrían un abastecimiento regular, en la práctica este resultado no es el más frecuente, particularmente para los primeros. Las razones varian en el caso de cada acuerdo. Si se toma como ejemplo el Acuerdo Internacional del Café2b, se podrá observar que la economía del sector se caracteriza por una tendencia a la sobreproducción y al subconsumo, lo que siempre tiende a deprimir los precios;

${ }^{23} \mathrm{Al}$ discutirse la Resolución 1721 (LIII) del Consejo Económico y Social de las Naciones Unidas, que acordó designar un grupo de expertos para estudiar el rol de las empresas multinacionales, se propuso que el estudio no se limitase a las empresas privadas sino que también comprendiera los consorcios públicos que operan en los paises en desarrollo. Véase la intervención del delegado de México, señor Muñoz Laredo, E/AC.6/SR. 586, 27 de julio de 1972, p. 3.

${ }^{2}$ Felipe H. Paolillo: "El comercio de Estado. Alternativa posible de la cláusula de la nación más favorecida en la estructuración del comercio internacional". En op. cit., Nota 21 supra.

${ }^{20}$ Sobre los acuerdos de productos básicos y otros ensayos de estabilización de los precios, Johnson, op. cit., Nota 16 supra, pp. 136-162.

${ }^{20}$ Celso Lafer: "El Convenio Internacional del Cafe", Derecho de Integración, No 12, marzo 1973, INTAL, Pp. 111-135. 
además, por no tratarse de un producto básico de interés vital para sus consumidores, estos últimos se encuentran en una posición ventajosa para controlar el funcionamiento del sistema e imponer sus condiciones. Es cierto que dentro de un marco de simple oferta y demanda el resultado habría sido todavía más negativo, pero esto no significa que el acuerdo pueda considerarse como una respuesta definitiva. En otros casos como en el Acuerdo del Trigo ${ }^{27}$, si bien el esquema original también tendía a apartarse de la oferta y de la demanda, con posterioridad ha evolucionado hacia una mayor libertad en el funcionamiento del sector, precisamente por convenir así al interés de los países desarrollados, que en este caso son a la vez los mayores productores. ${ }^{28}$

La experiencia emanada de los acuerdos sobre productos básicos, permite sostener que ellos atenúan el impacto derivado del sistema clásico pero no logran sobreponerse enteramente al mismo. Pero, aun asumiendo que clichos acuerdos fueran una alternativa viable, subsistiría siempre el problema de su sectorialidad; en efecto, aun cuando las relaciones económicas entre los países industrializados y los países en clesarrollo se estructuran de manera perfecta sector por sector, ello no bastaría como solución por cuanto los primeros continuarían ejerciendo una posición dominante sobre el conjunto, en tanto que los segundos se verían por general limitados a actuar dentro del sector específico impuesto por su condición de monoproductor. De ahí que, como se verá más adelante, el enfoque de una reestructuración necesariamente debe ser global.

\section{Preferencias comcrciales}

El tercer mecanismo de interés, siempre desde el punto de vista de la introducción de principios diferentes a los tradicionales, es el de las preferencias comerciales ${ }^{29}$. La principal característica del sistema de preferencias es que él reconoce como principio básico el del tratamiento diferencial en función del grado de desarrollo económico, con lo cual las partes de menos desarrollo ya no se ven sujetas en

${ }^{27}$ Felipe H. Paolillo: "Los acuerdos internacionales sobre el trigo". En op. cit., Nota 21 supra.

${ }^{28}$ Recientemente Estados Unidos se ha retirado de otros dos acuerdos orientados a resolver los problemas de precios: el del azúcar y el coco. Erb, loc. cit., Nota 2 supra, p. 37.

${ }^{20}$ Para un útil análisis de las preferencias, en la perspectiva del ordenamiento del comercio internacional, G. P. Verbit: "Preferences and the public law of international trade: the end of most-favoured-nation treatment?". Hague Academy of International Law, Colloquium, 1968: International Trade Agreements, Sijthoff, 1969, pp. 19-84. 
su comercio a normas iguales $\lambda$ las que se aplican a los países industrializados; en otras palabras, los países industrializados se ven discriminados, a fin de otorgar un tratamiento preferente para el comercio de los países en desarrollo. De esta manera, la desigualdad económica de las partes se regula por normas jurídicas también desiguales, en beneficio de la parte más débil, que incrementa así su capacidad competitiva. Ello de por sí configura una importante innovación respecto del sistema de la cláusula de la nación más favorecida, el cual, como se ha indicado, sujeta a las partes a un tratamiento jurídicamente igual, sin tomar en consideración su diferente grado de desarrollo.

Estas características del sistema de preferencias determinan otras consecuencias de importancia. Una de ellas es que el costo de la industrialización en el producto de que se trate, se traslada del país en desarrollo al país desarroliado que otorga la preferencia ${ }^{30}$, al menos en la medida en que este último estará pagando un precio mayor que aquel que habría pagado en un esquema de libre competencia. Puede observarse que en este caso se produce una diferencia fundamental respecto del sistema de protección del "infant industry", que era la concesión máxima hecha por el sistema clásico en favor de la industrialización de los países en desarrollo, puesto que en esta última situación el costo de la industrialización recala enteramente en el propio país en desarrollo ${ }^{31}$. De lo anterior, surge otra consecuencia igualmente significativa: las preferencias comerciales involucran una redistribución del ingreso al nivel internacional, en parte por la absorción del costo de industrialización que hace el país desarrollado que otorga la preferencia, y en parte porque se genera una corriente de ingresos hacia los países en desarrollo beneficiarios de la preferencia que de otro modo se habría radicado en los propios países industrializados de mayor capacidad competitiva.

No obstante la importancia de los principios inherentes al sistema de preferencias, éste no alcanza a una modificación de fondo del sistema económico internacional. Desde luego, su carácter transitorio no permite pensar en él como una solución permanente y probablemente son pocos los paises en desarrollo que podrían aprovechar oportuna y globalmente sus ventajas ${ }^{32}$. Por otra parte, este sistema no precave la tendencia alcista de los precios de los productos industriales que los paises en desarrollo deben siempre importar, con lo

${ }^{80}$ Ibid., pp. 32-33.

"Johnson, op. cit., Nota 16 supra, p. 181.

${ }^{32}$ Por esta razón se ha propuesto que el esquema de preferencias vaya acompañado de otras medidas adicionales que incrementen la capacidad competitiva de los países en desarrollo. Véase Erb, loc. cit., Nota 2 supra, p. 36. 
cual en una medida importante continúan pagando el costo de la inflación que emana de los países desarrollados, lo que eventualmente puede llegar a deteriorar seriamente el beneficio derivado de las preferencias. Además, debe tenerse presente el impacto de la política de inversiones extranjeras en el sistema de preferencias, en cuyo contexto debe evaluarse el rol de las empresas multinacionales y los beneficios que ellas están en condiciones de obtener ${ }^{33}$. Si a ello se agregan todavía los problemas que emanan de las preferencias recíprocas, la discriminación por bloques regionales o por sectores y el hecho de que el rol contralor del sistema continúa fuertemente concentrado en los países desarrollados, se podrá apreciar que este mecanismo dista mucho de ser la solución perfecta. ${ }^{34}$

La conclusión que cabe extraer de lo expuesto es que si bien ninguna de estas nuevas estructuras del comercio internacional logra superar satisfactoriamente las deficiencias del sistema clásico, ellas han logrado introducir en el medio internacional principios relevantes que, insertados en un marco apropiado, pueden ser parte de una solución más permanente. Todos estos principios tienen en común el que se apartan del esquema de libre competencia irrestricta; pero mientras algunos de ellos procuran conciliar los intereses de productores y consumidores sobre la base de la reglamentación del mercado, otros enfatizan el tratamiento diferencial en función del grado de desarrollo y la redistribución del ingreso en el plano internacional.

Dos hechos fundamentales contribuirían a hacer evidente la necesidad de una reestructuración general: el impacto de las empresas multinacionales en el sistema internacional y la presión del tercer mundo constituido en bloque; a la vez, estos dos factores precipitarían la crisis que venía gestándose. Estos aspectos del problema son los que se examinan a continuación.

\section{4. - LAS EMPRESAS MULTINACIONALES: EL CUESTIONAMIENTO DEL SISTEMA CLÁSICO POR LOS PAÍSES INDUSTRIALIZADOS}

\section{El sistema económico tradicional dio nacimiento al fenómeno de}

${ }^{88}$ El sistema de preferencia puede determinar la transferencia de producciones industriales de los países desarrollados a los paises en desarrollo, para aprovechar asl el mayor margen de competitividad que recibe el pais beneficiario, lo que se ve facilitado por la gran movilidad del capital, tecnología y capacidad gerencial. Véase Johnson, op. cit., Nota 16 supra, pp. 194-195.

"Sara un examen de los argumentos relativos a las ventajas y desventajas de las preferencias, ibid., pp. 164-206. 
las empresas multinacionales, que alcanzan su apogeo en la postguerra, bajo los acuerdos de Bretton Woods. Los progenitores de estas empresas fueron, por una parte, el principio de la libertad del mercado internacional y de la libre competencia irrestricta $y$, por otra parte, el fomento masivo de la inversión privada extranjera, que a su vez fue la consecuencia de la enorme concentración de capital que los países industrializados lograron sobre la base de los beneficios obtenidos en el funcionamiento de ese sistema. ${ }^{35}$

Las empresas multinacionales ciertamente fueron consecuentes con los postulados en que descansaba el sistema clásico. En primer término porque operan en el mercado internacional en función de las ventajas comparativas y de la división internacional del trabajo. En segundo lugar porque se fundamentan en la libre circulación de capitales, bienes y servicios. En tercer lugar, porque son un vehículo que garantiza el acceso no discriminado de los bienes industriales y de la tecnología a los mercados internacionales. Y en cuarto lugar, porque también ha sido el vehículo que en muchos casos aseguró el libre acceso de los países industrializados a las materias primas del mundo en desarrollo.

Sin embargo, en la medida en que las empresas multinacionales se desarrollaron, su impacto en el propio sistema internacional excedió de todo lo previsible. El enorme volumen de comercio, inversión y producción concentrado en sus manos ${ }^{3 \theta}$, unido al hecho de operar con flexibilidad en muchos países ${ }^{37}$, no solamente las transformó en poderosos conglomerados económicos sino que además facilitó el que adquirieran autonomía respecto de los propios Esta-

${ }^{85}$ Entre 1960 y 1971 , el valor en libros de la inversión directa de Estados Uni. dos aumentó de 33.000 a 86.000 millones de dólares; Inglaterra duplicó su inversión, pasando de 12.000 a 24.000 millones; Japón aumentó en 15 veces, pasando de 300 a 4.500 millones; Alemania registró un aumento de 10 veces. Naciones Unidas: Las corporaciones multinacionales en el desarrollo mundial, ST/ECA/190, 1978 , p. 8.

${ }^{30} \mathrm{La}$ producción internacional, esto es aquella sujeta a control o decisión extranjera, ha sido estimada para 1971 en 330.000 millones de dolares y, según otros cálculos, llegaria hasta 450.000 millones; en cualquier caso ella ya supera las exportaciones totales de los países de economía de mercado, que alcanzaron a 310.000 millones de dólares. Ibid., p. 14. Igualmente se ha estimado que en la década de 1980 , el $75 \%$ del comercio mundial y-de la producción industrial estará en manos de aproximadamente 300 empresas multinacionales. Detlev F. Vagts: "The Global Corporation and International Law", The Journal of International Law and Economics, Vol. 6, 1972, p. 249.

${ }^{37}$ Estados Unidos y Europa Occidental cuentan con 7.276 empresas matrices, con un número mínimo de 27.300 filiales en el extranjero. 3.357 de esas matrices tienen filiales en un soblo país extranjero; 3.241 en 2 y hasta 9 países; 501 en 10 a 19 países; y 177 tienen filiales en más de 20 países. Naciones Unidas, op. cit.. Nota 35 supra, p. 131. 
dos, inclusive respecto de aquellos donde habían tenido su origen ${ }^{38}$. De esta manera, aun cuando las diversas partes componentes de la empresa puedan estar ubicadas físicamente en el seno de diversos Estados, el conjunto de la empresa llegó a guiarse por intereses y políticas propias, diferentes de aquellas de los diversos Estados en que actúa.

Por otra parte, su movilidad internacional permitió que en muchos casos la empresa actuara como productor y consumidor al mismo tiempo, pero establecida en diferentes mercados. Así, por ejemplo, pudo producir materias primas en un país en desarrollo y consumirlas en un país desarrollado, aplicando siempre la teoría de las ventajas comparativas pero ya no medidas en términos del interés de un país frente a otro sino en términos de la estrategia de producción de una misma entidad. La incidencia de este fenómeno en el sistema de comercio internacional, y quizás en el propio sistema de preferencias, no debe pasar desapercibida ${ }^{30}$. Esta situación abrió a la vez la posibilidad de que la empresa actuara ya sea en consonancia con el interés de los países desarrollados, o ya sea en consonancia con el interés de los países en desarrollo, en función de su propia conveniencia en una coyuntura determinada.

$\mathrm{Si}$ a lo anterior se agregan, entre muchos otros, los factores tecnoIógicos, las posibilidades de manipulación de precios en las transacciones internas de la empresa y las restricciones que la política global de la empresa puede imponer a la política local de sus componentes, se podrá apreciar que esta nueva estructura unitaria trasciende las fronteras políticas tradicionales con amplitud ${ }^{40}$. Lo paradojal de este fenómeno es que, por grande que haya sido su impacto en los países en desarrollo, lo ha sido todavía mayor en los propios países desarrollados, por cuanto la dimensión de sus mercados, su poder consumidor y su abundancia de capitales constituyen el escenario natural para la acción de una entidad de la proporción de las empresas multinacionales. ${ }^{41}$

Cuando los países desarrollados percibieron su dificultad, y en ocasiones su impotencia, para controlar la acción de dichas empresas; cuando sintieron los efectos de la inversión por ellas realizada en el extranjero sobre sus balanzas de pago; cuando su propia capa-

"Véase en general el conocido estudio de Raymond Vernon: Sovereignty at Bay: the multinational spread of U.S. enterprises, New York, Basic Books, 1971.

${ }^{8}$ Naciones Unidas, op. cit., Nota 35 supra, pp. 63-64.

'Ibid., pp. 39 et seq.

«De acuerdo a cifras de 1966, 15.128 filiales de empresas norteamericanas se encontraban establecidas en países desarrollados de economía de mercado, lo que representa un $65 \%$ del total. 7.718 filiales operaban en países en desarrollo, lo que representa un $33,2 \%$. Ibid., p. 136. 
cidad competitiva se vio resentida por la capacidad que las propias empresas habian creado en otros países; cuando sus fuerzas laborales fueron afectadas por el recurso de las empresas al trabajo más barato de otros paises; cuando sus balanzas comerciales conocieron el signo negativo; cuando sus propias monedas cayeron en el frenesí de la especulación por parte de las empresas que cambiaban sus reservas; cuando sus relaciones políticas internacionales se hicieron difíciles por la acción de empresas multinacionales en países extranjeros; o cuando su propia subsistencia como sociedades industriales se vio amenazada por la crisis de la energía, y el incógnito rol de las empresas petrolíferas, entonces se inició el cuestionamiento de las virtudes de la división internacional del trabajo y de la libertad irrestricta del mercado internacional. ${ }^{42}$

La reacción de los países desarrollados ante este fenómeno es todavía elemental. Hasta ahora ha consistido en medidas de defensa de la balanza de pagos y otras medidas auxiliares en diversos planos. Igualmente han surgido numerosas propuestas sobre aplicación extraterritorial de determinadas legislaciones, códigos de buena conducta, revelación de información, procedimientos de consulta y negociación y otras iniciativas, ninguna de las cuales alcanza a atacar el fondo del problema ${ }^{43}$. En realidad, recién se ha comenzado a apreciar la magnitud del problema y no hay todavía un criterio formado acerca de cómo encararlo.

Ello explica que la atención se haya concentrado únicamente sobre las empresas multinacionales, y sobre ellas haya recaído la culpa de los efectos adversos que se observan en el sistema económico internacional. Pero, en honor a la verdad, este es un enfoque limitado e injusto. Como se explicó anteriormente, las empresas multinacionales han sido perfectamente consecuentes con los postulados del sistema tradicional, habiéndolos llevado a su más acabada expresión; en consecuencia, la solución no radica en controlar solamente lo que es una manifestación del problema, sino que se hace necesario atacar el problema mismo, esto es, reestructurar los principios en que descansa ese sistema económico, con lo cual no sólo se habrá controlado una manifestación individual simbolizada en la empresa multinacional, sino se habrán precavido también un sinnúmero de otras expresiones.

En todo caso, el impacto de la empresa multinacional ha sido lo que ha permitido la primera aproximación de los países desarrolla-

\footnotetext{
"Para un resumen de los problemas suscitados por las empresas multinacionales en sus países de origen y citas bibliográficas, ibid., pp. 56-57.

- ${ }^{3}$ Para un examen de las diversas medidas propuestas en este plano, Francisco Orrego Vicuña: "El control de las empresas multinacionales", Foro Internacional, El Colegio de Méxíco, Ne 53, julio-septiembre 1973, pp. 106-128.
} 
dos a una toma de conciencia acerca de las deficiencias del sistema tradicional. En la medida en que este proceso se acentúe irán madurando las condiciones necesarias para la reestructuración; la presión ejercida por el tercer mundo, que se examinará a continuación, es otro de los factores que más poderosamente ha contribuido a esta maduración.

5. - LA CONSOLIDACIÓN DEL TERCER MUNDO COMO PODER REIVINDICATIVO

En el curso de la postguerra los países en desarrollo comenzaron en forma progresiva, y cada vez más acelerada, a tomar conciencia del alcance real que para ellos tenía el sistema económico internacional. Con motivo de la discusión de la Carta de La Habana ya se observan los primeros planteamientos sistemáticos destinados a corregir la situación de desventaja que los afectaba ${ }^{44}$; y, con motivo del proceso de descolonización, lo que en un comienzo fue la posición de un número limitado de países de América y Asia, se transformó gradualmente en la concepción común de un conglomerado enorme de países. De la misma manera, lo que en un comienzo se limitó a planteamientos sobre problemas determinados del comercio internacional, paulatinamente fue extendiéndose al conjunto del espectro comercial, abarcando además el ángulo de las inversiones extranjeras, las reivindicaciones de las riquezas marítimas y otros muchos aspectos, todo lo cual culminó en definitiva en el cuestionamiento general del sistema económico internacional.

E1 proceso de consolidación institucional del tercer mundo se ha fortalecido notablemente en la última década. Desde las primeras acciones comunes en el seno de la Asamblea General de Naciones Unidas, caracterizadas más bien por un enfoque intuitivo y casuístico que por un análisis detenido, hasta la primera UNCTAD, donde ya emergió una presión coherente, y pasando por un número importante de experiencias como las reuniones de países no alineados, la acción del grupo de los 77 o la actividad de grupos regionales, el tercer mundo aprendió a conocerse y adquirió la capacidad de desarrollar una actitud concertada y sostenida. ${ }^{45}$

No obstante este proceso de coordinación creciente, el tercer mundo dista aún mucho de constituir un grupo uniforme que descanse

"Véase Nota 19 supra.

${ }^{46}$ Felipe H. Paolillo: "La Estrategia del tercer mundo: Apuntes sobre la solidaridad de los paises en desarrollo en su lucha intexnacional por reivindicaciones económicas". En op. cit., Nota 21 supra. 
en intereses plenamente coincidentes. Los diferentes grados de desarrollo que existen en el tercer mundo, en que la brecha que separa a los más pobres de los más prósperos es todavía mayor que la que separa a estos últimos de los países desarrollados; los diferentes intereses que los paises tienen como productores, en ocasiones contradictorios y competitivos; las diferencias de sistema político y cultural son, entre muchos otros, factores que tienden a dispersar la acción y estrategia. Ello explica que más allá de los grandes principios básicos, el tercer mundo todavía no haya logrado un acuerdo sobre cómo organizar o llevar a la práctica sus reivindicaciones específicas, salvo en sectores determinados.

Sin embargo, es un error deducir de lo anterior la conclusión de que la dispersión del tercer mundo es más fuerte que sus tendencias de unión. En este aspecto radica precisamente el juicio equivocado de los países desarrollados, que adoptaron la estrategia de prescindir del clamor del tercer mundo, ya sea no discutiendo los problemas o cuando forzados a ello ignorando las recomendaciones ${ }^{40}$, en la creencia de que los factores de dispersión impedirían la efectividad de las reivindicaciones y de que siempre sería posible un entendimiento bilateral, o a lo más regional, en función de los problemas concretos de interés para los países desarrollados. Tal estrategia pudo dar frutos en el corto plazo, pero no en el mediano ni en el largo. La prueba está en que la posición del tercer mundo se ha ido radicalizando, como consecuencia de no haber logrado soluciones satisfactorias respecto de ninguna de sus reivindicaciones básicas. Si se comparan, por ejemplo, los primeros planteamientos ante la Asamblea General con aquellos realizados en UNCTAD I, o estos últimos con los de las recientes conferencias de los países no alineados, se podrá apreciar claramente la evolución. Igual tendencia se manifiesta en otros planos, como por ejemplo el de las inversiones extranjeras o el del derecho del mar.

La dispersión del tercer mundo es real, pero más real todavía es su nítida percepción de los problemas inherentes al sistema económico internacional y, en particular, del hecho de que éste sólo les asigna un rol periférico sin tomar en cuenta sus necesidades. Esta percepción ha llegado a transformarse en la filosofía común del tercer mundo y, como tal, es aplicada en todas las coyunturas que se presentan, que es lo que explica una unidad conceptual permanente $\mathrm{y}$, aun cuando todavía limitada a lo básico, lo suficientemente fuerte

${ }^{\circ}$ El número de votos en contra y de abstenciones por los paises desarrollados, respecto de los Principios Generales y Principios Especiales, en la I UNCTAD, es lo suficientemente revelador al respecto. Cuadros en Johnson, op. cit., Nota supra, pp. 252-253. 
para perseverar más allá de las derogaciones que significan los factores de dispersión.

\section{El control de las materias primas}

Hasta hace poco tiempo, el tercer mundo había puesto el énfasis principal de su estrategia en presionar por reivindicaciones que deseaba obtener de los países desarrollados, principalmente en lo que se refiere al acceso no discriminatorio a sus mercados y al logro de precios justos; pero no había utilizado su propia potencialidad económica para fortalecer su posición en el sistema internacional. Este es el periodo que podría llamarse de las reivindicaciones pasivas. Como consecuencia de no haber sido escuchados sus planteamientos, se inicia en la década de 1960 el período que podría llamarse de las reivindicaciones activas, que alcanza madurez en la década de 1970 y aún se encuentra en pleno desarrollo. Este período se caracteriza por un tercer mundo que aprende e inicia el aprovechamiento de su propia fuerza, ya no confiando en lo que los países desarrollados buenamente estén dispuestos a conceder, sino que adoptando las medidas consideradas adecuadas para lograr sus fines reivindicativos.

Dado el hecho de que la mayor riqueza económica del tercer mundo son sus materias primas, la estrategia activa lógicamente se concentró en el control de las mismas. Sin perjuicio de ello, factores tales como el peso de su enorme población, su incidencia en el esquema político mundial y otros coadyuvaron a que tal estrategia alcanzara un grado considerable de eficacia. El primer hecho visible es que el tercer mundo decidió no seguir permitiendo el libre acceso de los países desarrollados a sus materias primas, en los términos que lo había impuesto el sistema económico tradicional y, como fruto de éste, la operación de las empresas multinacionales. Esta decisión ni siquiera fue el producto de una acción concertada, pudiendo decirse que emergió en forma casi espontánea y simultáneamente en diferentes lugares, derivándose de la percepción común que se indicó. En algunos casos ello ha tomado la forma de legislación sobre control de inversiones extranjeras, en otros la forma de asegurar una participación del Estado y, en los más extremos, la forma de una nacionalización directa o indirecta ${ }^{47}$. En todo caso, es

*La nómina de expropiaciones y otros actos que han afectado propiedades norteamericanas en el extranjero, es demostrativa de esta tendencia. En el período 1960-1971, 34 países aplicaron medidas de este tipo: 9 en América Latina, 15 en Africa, 5 en el Medio Oriente y 5 en Asia. Véase: Department of State: "Report on nationalization, expropriation, and other taking of U.S. and certain foreign property since 1960", 30 de noviembre de 1971. En International Legal Materials, Vol. XI, No I, January 1972, pp. 84-118. 
incuestionable que el acceso de los países desarrollados a las materias primas es hoy día infinitamente más condicionado y controlado que lo que era al comienzo de la postguerra, tendencia ésta que se acentuará cada vez más en los próximos años.

Más importante todavía es la tendencia de los países en desarrollo a controlar el conjunto de las fuentes de abastecimiento de una determinada materia prima, mediante la utilización de los acuerdos de productores. De los diversos acuerdos de productores que se han ensayado ${ }^{48}$, el más célebre es por cierto el de la Organización de Países Exportadores de Petróleo (OPEC), que aprovechando las particulares condiciones de la economía del petróleo, caracterizada por un fenómeno de subproducción frente a uno de sobreconsumo, ha marcado un hito definitivo en la evolución de la estrategia del tercer mundo. En efecto, en este caso se puede apreciar con claridad la diferencia entre el periodo de reivindicaciones pasivas y el de reivindicaciones activas. Mientras en el primero OPEC procuró conseguir los mejores precios y condiciones de explotación sobre la base de negociar con las empresas multinacionales petrolíferas ${ }^{49}$, en el segundo adoptó por sí misma las medidas consideradas adecuadas para el fin perseguido. El resultado no solamente demostró la factibilidad de regular la producción y de aumentar las utilidades, sino además demostró la vulnerabilidad de los países desarrollados, que fueron precipitados a una crisis sin precedentes.

Lo paradojal de la experiencia de los acuerdos de productores es que ellos también son el fruto del sistema económico tradicional y de sus postulados de la libre competencia, oferta y demanda, y ausencia de restricciones. La única diferencia es que esta vez la posición dominante ha sido, quizás por primera vez, detentada por países en desarrollo. La concertación del acuerdo no es ni más ni menos que la formación de un cartel, del mismo tipo que por años organizaron los productores de bienes industriales al amparo de la ausencia de controles internacionales ${ }^{50}$. $\mathrm{El}$ aumento de precios constituye el aprovechamiento de una coyuntura de oferta y demanda, que tampoco se diferencia de las técnicas utilizadas por los países desarrollados. La discriminación en el acceso al producto es equivalente a la tradicional discriminación sufrida por las exportaciones del tercer mundo. El uso político de la coyuntura tampoco carece de precedentes.

\footnotetext{
«s Para una descripción sumaria de los principales acuerdos de productores, Hans Bachmann: The External Relations of Less-Developed Countries, Praeger, 1968 , pp. 251-253.

"Para una cronología de estas negociaciones, Isidro Morales Paul: "La Organización de Pafses Exportadores de Petróleo (OPEC) ". En op. cit., Nota 21 supra.

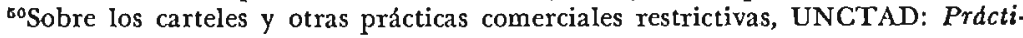
cas comerciales restrictivas, TD/122/Supp. 1, 7 de enero de 1972.
} 
Inclusive cabe observar que el resultado del proceso en su conjunto es idéntico al generado por el sistema tradicional: la concentración cle enormes cantidades de capital y prosperidad en manos del beneliciario que cletenta la posición dominante ${ }^{51}$, frente al deterioro gradual de la posición de la contraparte.

Por otra parte, es curioso observar que las reacciones generadas por el fenómeno son también idénticas a las del sistema tradicional, pero invirtiendo el rol de sus actores. El interés de los países procluctores ha sido llegar a entendimientos bilaterales con los consumidores, técnica a través de la cual se pueden obtener mayores ventajas para los primeros ${ }^{52}$; se recordará que éste fue el sistema que prevaleció hasta Bretton Woods y en una medida importante continuó operando hasta hoy. En cambio, el interés de los países afectados por el esquema ha sido el de armonizar su acción para una defensa común, dentro de lo cual también se observan factores de dispersión ${ }^{53}$. De esta manera, por lo menos en el sector a que se refiere el acuerdo, el tercer mundo ha aprendido de los países desarrollados cómo obtener el máximo beneficio del sistema tradicional; y estos últimos están aprendiendo del tercer mundo la estrategia para sus reivindicaciones.

Si bien es claro que el impacto de OPEC se ha debido en gran medicla a las particulares características de la economía del petróleo y a la coyuntura energética de los países desarrollados, no cabría subestimar su incidencia en la estrategia general del tercer mundo. Desde luego es previsible que esta experiencia se repetirá en todos aquellos sectores que representan un interés sustancial para los países desarrollados, esto es, en todos los casos en que el tercer mundo controle materias primas vitales. Como ejemplo de ello puede mencio-

"En el caso de los paises miembros de OPEC, se ha estimado que al precio dc mercado de 8 dólares por barril de petróleo, obtendrán en 1974 un ingreso de 85.000 millones de dólares. La magnitud de csta cifra es particularmente impresionante cuando se tiene presente que en 1970 los países en desarrollo, excluyendo los cxportadores de petróleo, sólo obtuvieron ingresos cle 47.431 millones de dólares por concepto de exportaciones de bienes y scrvicios, csto es, la mitad del ingreso de OPEC. Para 1980 el ingreso de OPEC, depencliendo de los precios, demanda y producción, pucde llegar a 150.000 milloncs de dólares y hasta 200.000 millones; ello equivaldria a la mitad de las exportaciones actuales de los pafses desarrollados de cconomia de mercado.

"2Como ejemplo de esta tendencia puede scĩalarse la advertencia hecha por el Ministro de Petrólcos de Arabia Saudita, Zaki Yamani, al Japón, acerca de los serios problemas que podrian afcctar a cste último si $\mathrm{cn}$ vez de concertar un arreglo vilateral procedia a actuar de acuerdo con otros pafses consumidores. The Was. hington Post, 29 de enero de 1974.

${ }^{53}$ Como ejemplo de esta otra tendencia pucde señalarse la conferencia de países consumidores de petróleo, convocada por el gobierno de Estados Unidos para reunirse en febrero de 1974 , y la cautelosa actitud asumida para los participantes. 
narse desde ya el caso de la bauxita -materia prima del aluminio-, cobre, estaño y goma natural, en que pocos países en desarrollo controlan la totalidad o un gran porcentaje de los abastecimientos ${ }^{54}$; la lista podría ampliarse sucesivamente, debido al hecho de que la mayoría de los países desarrollados son importadores netos de minerales ${ }^{55}$. Por otra parte, también es previsible la formación de acuerdos intersectoriales entre productores de materias primas que inciden en productos competitivos, como por ejemplo el cobre y la bauxita, con lo cual se llegaría al control de todos los conductores de electricidad. Incluso es posible que el esquema llegue a abarcar en un momento dado al conjunto de las materias primas. ${ }^{56}$

El control progresivo de las materias primas por otra parte del tercer mundo ha alterado fundamentalmente el funcionamiento del sistema económico tradicional, por cuanto éste ya no asegura el libre acceso a dichos productos, que era uno de los objetivos de la división internacional del trabajo. En la misma medida la propia movilidad de las inversiones extranjeras ha sido también afectada. Ello revela cómo el cuestionamiento del sistema tradicional por los países en desarrollo, se ha traducido en resultados efectivos. A su vez estos resultados han sido posibles debido a un cambio profundo de circuntancias: lo que durante muchos años fue considerado una riqueza inagotable, abundante y barata, son hoy día materias primas escasas y consecuentemente más caras.

Sin embargo, estos resultados parciales ni con mucho logran solucionar el conjunto de problemas derivados del sistema tradicional para los países en desarrollo, que se han expuesto anteriormente. En realidad, apenas logran una mejoría relativa en el aspecto específico que se refiere a los precios de las materias primas. $\mathrm{E}$ incluso en este aspecto se presenta el problema de que, así como el abuso del sistema tradicional por parte de los países desarrollados generó consecuencias que hoy comienzan a afectarlos adversamente, también

${ }^{84}$ Los mayores proveedores de bauxita son Guinea, Australia, Guyana, Jamaica y Surinam; una conferencia de productores ha sido convocada por Guinea en febrero de 1974. Cuatro países controlan el abastecimiento del $80 \%$ del cobre mundial y se encuentran organizados en el Consejo Intergubernamental de Países Exportadores de Cobre (CIPEC). Dos países controlan el abastecimiento del $70 \%$ del estaño. Cuatro países controlan la mitad de la producción de goma natural. The Washington Post, 14 de enero de 1974.

${ }^{8} \mathrm{E}$ l déficit en la importación de minerales por Estados Unidos alcanza a 6.000 millones de dólares anuales. Ibid. Para 1985 se estima que Estados Unidos deberá importar la totalidad de su consumo de cromo, cobalto, manganeso y estaño, así como altos porcentajes de aluminio, cobre, hierro, níquel y otros minerales. Overseas Development Council, op. cit., Nota 2 supra, p. 137.

-Para un examen de las principales tendencias de este plano, Overseas Development Council, op. cit., Nota 2 supra, pp. 13-14. 
una utilización exagerada de la coyuntura por parte de los pafses en desarrollo podría dar lugar a efectos contraproducentes, como ejemplo de lo cual puede mencionarse el caso de una eventual recesión mundial provocada por los precios del petróleo.

La presión del tercer mundo ha sido lo suficientemente sostenida como para crear las condiciones apropiadas para considerar la reestructuración del sistema económico internacional sobre bases que tomen en cuenta la necesidad legítima de sus dos segmentos básicos. A su vez ha sido uno de los factores inductivos más poderosos de la crisis actual de ese sistema. Los propios países desarrollados han podido percibir con mayor claridad los inconvenientes del sistema tradicional, a la luz de la presión del tercer mundo y en particular de la crisis de la energía, lo que unido al problema de las empresas multinacionales anteriormente mencionado, ha acentuado la madurez necesaria para proceder a la reestructuración.

\section{6. - LA CONFIGURACIÓN DE LA CRISIS GENERAL}

Los elementos de juicio que se han expuesto revelan que el sistema tradicional ya no es adecuado ni siquiera para las necesidades de los países desarrollados, y menos aún para los países en desarrollo. Los primeros ya no gozan de la libertad de acceso a las materias primas en los términos irrestrictos que caracterizaron el pasado; los precios de las mismas han aumentado proporcionalmente su escasez, con lo cual la ventaja comparativa de la producción de bienes industriales se ha visto en cierta medida deteriorada; consecuencia de ello es que la división internacional del trabajo ya no determina necesariamente un flujo permanente de riqueza y muchos de los ingresos deben destinarse a la compra de bienes primarios en el tercer mundo; de esta manera, el acceso no discriminado de los bienes industriales al mercado internacional en cierto modo se resiente en su rol de generador neto de utilidades. Incluso muchos de los beneficios que todavía subsisten tienden a radicarse en las empresas multinacionales, que en muchos casos ya no pueden identificarse enteramente con el interés o beneficio de sus países de origen. Ciertamente sería una exageración sostener que el cuadro que se viene describiendo es absoluto o que ya se da en forma general, pero no menos cierto es el hecho de que importantes tendencias y comprobaciones prácticas apuntan en ese sentido.

Respecto de los países en desarrollo ya se han indicado con anterioridad los inconvenientes derivados del sistema tradicional y el hecho 
de que la mejoría relativa recientemente registrada por el precio de algunas materias primas no satisface sino uno de los muchos problemas todavia presentes, e incluso sobre bases cuya estabilidad o permanencia es difícil de predecir frente a la enorme capacidad de innovación tecnológica de los países desarrollados. De esta manera, siempre subsisten problemas como el precio de los bienes industriales, que siempre deben importarse; como el acceso de su propia producción a los mercados desarrollados; como las prácticas restrictivas de las empresas multinacionales o corporaciones estatales; como la ausencia de redistribución internacional, agravada por la tendencia de los países desarrollados a restringir su ayuda bilateral y multilateral como consecuencia de tener que destinar mayores sumas a la compra de algunas materias primas; y, en general, como lo que se ha descrito ser su rol periférico.

Este complejo cuadro es el que explica las numerosas crisis parciales que se registran en el medio internacional, todas las cuales, como se ha indicado, son manifestaciones de una crisis general subyacente motivada por la inadecuación de los principios del sistema tradicional ante la realidad contemporánea. La tendencia que todavía prevalece es la de buscar soluciones parciales y ad hoc respecto de cada crisis individual. Así, por ejemplo, se busca atacar el problema de las empresas multinacionales sin atender a las causas que lo genera; o se busca solucionar la crisis de la energía sobre la base de un entendimiento entre productores y consumidores en ese sector específico, sin relacionarlo tampoco con sus causas, que son mucho más profundas y generalizadas y que exceden del marco de ese sector individual.

Este enfoque parcial sólo contribuye a agravar la situación y a demorar las soluciones de fondo. En efecto, asumiendo por ejemplo que se lograra un acuerdo mutuamente satisfactorio para las partes en el caso de la energía, él ciertamente no impide que el problema vuelva a repetirse sucesivamente en otros sectores; tampoco el hecho de asegurar la prosperidad de un número reducido de países productores tiene incidencia en el problema general que afecta al tercer mundo o a los productores de otras materias primas. Por otra parte, ello siempre permitiria a los países desarrollados controlar el conjunto de los sectores que conforman el sistema económico, por cuanto como se indicó anteriormente los países en desarrollo sólo estarían en condiciones de actuar en el sector específico impuesto por su calidad de monoproductor, con lo cual tampoco se solucionaría el problema de una participación equitativa en la conducción del sistema, que es una de las necesidades derivadas de la realidad contemporánea.

Igual fenómeno sucede con las tendencias hacia un entendimiento 
F. Orrego Vicuña / Do la crisis de la energfa al concepto del patrimonio...

bilateral entre los países desarrollados y los países en desarrollo que producen materias primas de interés vital, esquema en el cual los primeros ofrecen industrializar a los segundos a cambio de un abastecimiento seguro ${ }^{57}$, pero cuyo impacto en una solución general es nulo. Incluso en el caso de llevarse este esquema a una escala regional se producirían los mismos problemas, con el agravante de que se afectaría seriamente la capacidad operacional del tercer mundo, el que se encontraría dividido en bloques ligados por un entendimiento con uno o más países desarrollados en la cúpula, tal como en un determinado momento pudo suceder en las preferencias regionales. Así como entonces se insistió en un esquema de preferencias generalizadas, también ahora corresponde insistir en un arreglo global, sobre todo ante el hecho de que una solución regional podría impedir los acuerdos sectoriales o intersectoriales de productores que exceden de ese marco, sin perjuicio de los impedimentos que puedan emanar para una acción general concertada del tercer mundo. Quizás, el enfoque bilateral o regional pueda concebirse como una etapa transitoria en el proceso de reestructuración global, por cuanto sería imposible pedirles a los países en desarrollo que se abstengan de todo arreglo en el intertanto, pero el objetivo final necesariamente debe ser un esquema global.

Cabe, en consecuencia, concluir que siendo la crisis que afecta al sistema económico internacional de carácter generalizado, la solución a que se llegue debe necesariamente ser de la misma naturaleza, como única manera de asegurar su estabilidad y su permanencia. Todo ello asumiendo que exista en la comunidad internacional el propósito de buscar soluciones racionales, ya que con frecuencia se revierte el argumento del empleo de la fuerza y de las represalias por parte de los países desarrollados, lo que no es improbable si acaso no se encuentra una solución pacífica en corto plazo, aun cuando dichos métodos significarían el comienzo de una conflagración también generalizada. Pero asumiendo que esa racionalidad existe, cabe referirse a los lineamientos centrales de una reestructuración.

${ }^{67} \mathrm{El}$ caso más típico lo ofrece en este sentido Japón, país que a través de diversos proyectos ha destinado sumas cercanas a los 1.500 millones de dólares a varios palses productores de petróleo del Medio Oriente, sin perjuicio de proyectos en Indonesia y otros bajo consideración. The Washinglon Post, 3 de febrero de 1974. Similar política está siguiendo Alemania y Francia, entre otros países. 
El sentido de cualquier reestructuración que se intente, debe ser el de buscar una solución a las necesidades que se han hecho evidentes en una comunidad internacional caracterizada por su interdependencia. La más elemental de estas necesidades es evitar que los países en desarnollo continúen un proceso de empobrecimiento progresivo, frente a una prosperidad creciente de los países desarrollados; esta debe ser la base misma de una convivencia social pacífica. Ello supone que el sistema económico no solamente atienda a las necesidades de los países en desarrollo, sino especialmente que les reconozca una participación equitativa en su seno, lo que no excluye que también atienda a las necesidades legitimas de los propios países desarrollados.

Como se pudo apreciar, la libre competencia en que se fundamentó el sistema clásico no fue capaz de asegurar ese equilibrio, no porque el principio no sea adecuado, sino porque en realidad nunca fue aplicado con propiedad. Para que exista una competencia sana se requiere en primer término la abolición de todo tipo de prácticas restrictivas, pero el sistema clásico fue un solo conjunto de prácticas restrictivas. El régimen de competencia supone igualmente que quien detenta una posición dominante en la economía no abuse de ella, pero en el sistema clásico los países desarrollados usaron y abusaron de su posición dominante. En ambos vicios han incurrido también en muchas ocasiones las empresas multinacionales. Sobre todo una competencia sana exige de una protección especial a quienes por su debilidad no están en condiciones de resistirla, lo que supone el tratamiento preferencial y no una igualdad jurídica formal. Además de todo lo anterior, todo régimen de libre competencia requiere de otras medidas absolutamente indispensables: acceso no discriminatorio a los abastecimientos y al mercado, que es un principio fundamental para asegurar la igualdad de oportunidades; y redistribución del ingreso como manera de evitar la concentración del poder económico, por una parte, y como manera de garantizar el ingreso mínimo a los sectores de bajos ingresos, por otra parte.

Desde el momento en que ninguna de estas condiciones se cumplieron bajo el sistema clásico, con los resultados conocidos, ellas deben ser las que inspiren el sentido de su reestructuración, que no necesariamente debe descartar la competencia como uno de sus 
postulados, pero sí garantizar su correcta aplicación. Los recursos y riquezas de la comunidad internacional ya no pueden seguir considerándose ni administrándose como el patrimonio de los más poderosos; debe avanzarse hacia una etapa en que se le conciba como el patrimonio económico de la humanidad, respecto del cual todos los países tienen derechos y también obligaciones, dentro de un enfoque de equidad y en un marco que tome en cuenta las legitimas necesidades y aspiraciones de todos ellos ${ }^{58}$. Este concepto del patrimonio económico de la humanidad descansaría sobre los siguientes principios mínimos:

a) El acceso no discriminatorio. El principio del acceso no discriminatorio aseguraría a los países desarrollados el abastecimiento de las materias primas requeridas por su industrialización, ya no como un "libre" acceso sino como un acceso en igualdad de oportunidades, habida cuenta de la disponibilidad del producto y otros factores que razonablemente determinen la política de producción. De esta manera, junto con evitarse la depredación de las fuentes, se evitarán futuros embargos. Por ello tendría también una contrapartida: el acceso no discriminatorio de los productos de los países en desarrollo a los mercados de los países desarrollados dentro de un esquema de tratamiento preferencial que, en función de los diferentes grados de desarrollo, les permita razonablemente participar de la competencia internacional. De esta manera se llevaría a la práctica el objetivo de proteger al más débil. Dentro de este contexto, debe llegarse a un esquema de equilibrio de precios que guarde una cierta relación con el objetivo de garantizar un tratamiento equitativo para las partes. De la misma manera, el principio aseguraria el acceso de los países en desarrollo a los bienes industriales que necesiten.

b) La prevención de los efectos adversos. Este otro principio se dirigirfa, en primer término, a precaver las prácticas restrictivas, tanto de las empresas multinacionales como de las corporaciones estatales, gobiernos u otros, y ya sea que tengan su origen en países desarrollados o en paises en desarrollo, excepto en las medidas en que legítimamente correspondan a un grado de protección derivado de las necesidades económicas de un país o de un grupo de países. En función de este principio es que también se precavería y sancionaría el

${ }^{88}$ Un precedente de importancia en este sentido es la Declaración de Principios aprobada por la Resolución 2749 (XXV) de la Asamblea General de las Naciones Unidas, mediante la cual se declaró que los fondos marinos y oceánicos fuera de los límites de la jurisdicción nacional son "patrimonio común de la humanidad". 


\section{ESTUDIOS INTERNAGIONALES}

abuso de una posición dominante. Pero, más allá de las prácticas restrictivas, este mismo principio estarfa dirigido a asegurar que determinadas medidas, aun cuando lícitas, no se traduzcan en efectos adversos para la economía de uno, varios o la totalidad de los países, habida cuenta de su capacidad económica; así, por ejemplo, si el excesivo aumento de precio de un determinado producto, no originado en una manipulación sino en una situación de escasez, llegara a significar un drenaje en la balanza de pagos de un país sin que éste pueda razonablemente absorberlo, el principio operaría con miras a una corrección del problema ${ }^{50}$, sin que tampoco signifique afectar al productor en términos que excedan de lo razonable. Igual función desempeñaría el principio en caso de un descenso anormal de los precios, fluctuaciones monetarias graves u otras circunstancias que generen impactos mayores en la estabilidad económica.

c) La redistribución del ingreso internacional. El mecanismo clave de un nuevo sistema debe ser el de la redistribución del ingreso, en forma proporcional al beneficio obtenido. Como se indicó anteriormente, sólo de esta manera se puede garantizar que no haya una excesiva concentración del poder económico y que los sectores de más bajos ingresos alcancen un ingreso mínimo. A la vez, esta es la mejor garantía de estabilidad internacional y una norma de innegable justicia. Si bien el sistema de las preferencias comerciales involucra un grado de redistribución, ello no es suficiente; menos todavía lo es la ayuda internacional, ni siquiera en el caso que los países desarrollados cumplieran con el compromiso de transferir el uno por ciento de su producto bruto. Por esta razón es que debe llegarse a la creación de un sistema tributario internacional, que de alguna manera apropiada grave los ingresos derivados de la operación del sistema internacional o de otras fuentes, en forma proporcional y progresiva ${ }^{60}$; a su vez debería crearse un fondo internacional que canalice la redistribución a través de los medios adecuados. Un sistema de esta naturaleza no sólo sería aplicable a los países desarro-

\footnotetext{
${ }^{80}$ En 1972 los países en desarrollo importadores de petróleo tuvieron un gasto de 900 millones de dólares por este concepto; en 1979 subio a 2.300 millones; en 1974 alcanzará a 11.600 millones de dólares; y se estima que para 1980 estas cifras pueden fluctuar entre 25.000 y 38.000 millones de dólares. El impacto en la balanza de pagos es de gran magnitud. En 1970 la totalidad de los ingresos en moneda extranjera de los países en desarrollo, excluyendo los exportadores de petróleo, por concepto de exportaciones de bienes y servicios, flujos oficiales y privados, transferencias de pagos, líneas de crédito y derechos especiales de giro, sólo alcanzaron a 66.983 millones de dólares.

${ }^{\circ}$ Para diversas proposiciones relativas a esquemas tributarios parciales, James W. Howe and Mildred Weiss: "New financial Resources for the Poor Countries". En Overseas Development Council, op. cit., Nota 2 supra, pp. 54-55.
} 
llados y sus empresas, sino también a los propios paises en desarrollo en la debida proporción. De esta manera se aseguraría que cuando se produzcan grandes concentraciones de capital y riqueza en manos de algunos países en desarrollo, como consecuencia de coyunturas especiales u otras causas, también los demás países en desarrollo se beneficiarian mediante una redistribución razonable ${ }^{01}$, que en cualquier caso deberá cubrir por lo menos el margen de utilidades excesivas.

d) Institucionalización. La aplicación de los principios anteriores requerirá de la creación de instituciones que sean capaces de administrarlos y velar por su cumplimiento. Pero no deberá creerse que se necesita establecer una enorme maquinaria burocrática para este efecto. Bastaría con un cuerpo que tenga una capacidad de verificación eficiente y una gran capacidad de análisis, acompañada de un sistema de sanciones adecuadas. Los propios Estados Ilevarfan a la atención de este mecanismo los hechos o circunstancias que se estimen contrarios a los principios aludidos, proporcionando la información pertinente. Al mismo tiempo debería establecerse una obligación general de informar y proveer al mecanismo de los poderes necesarios para requerir información. Nada de lo anterior impedirfa un amplio procedimiento de consulta y negociación entre las partes involucradas, aun cuando también deberá establecerse un mecanismo para la solución de controversias económicas que ofrezca garantfas reales de imparcialidad. Eventualmente podría incluso pensarse en que el mecanismo que se establezca desempeñe la función de verificar la compatibilidad de las inversiones extranjeras que se le presenten con los principios aludidos, particularmente en cuanto a las condiciones de los contratos, y en caso de una conclusión afirmativa otorgar a esas inversiones una garantfa de inexpropiabilidad o a la posibilidad de un seguro internacional respecto de determinados riesgos.

Muchas de estas funciones, incluyendo la relativa al mecanismo de redistribución, podrían ser desempeñadas por los organismos internacionales nacidos de Bretton Woods, reestructurados en función del nuevo sistema económico internacional y por otros organismos dotados de la experiencia técnica necesaria. Debe, sin embargo, tenerse presente que el requisito imperativo para el adecuado funcionamiento de todo este sistema, es que los países en desarrollo tengan

'El Shah de Irán ha sugerido que los ingresos obtenidos por los patses productores de petróleo, que no puedan ser utilizados en esos mismos países, se destinen a algún mecanismo como un banco internacional para la ayuda a los países en desarrollo. Entrevista de la Revista Der Spiegel reproducida por The W'ashington Post, 3 de febreto de 1974. 
una participación equitativa en los mecanismos institucionales y decisorios, pues de lo contrario el sistema continuaría influido exclusivamente por los intereses de los países desarrollados.

La evolución de la sociedad internacional guarda una estrecha relación con lo que en el pasado caracterizó la evolución de muchas sociedades nacionales.

La revolución industrial del siglo xux, junto con generar una enorme prosperidad, dio lugar a fuertes tensiones sociales como consecuencia de la inadecuada distribución del ingreso en la sociedad, la concentración del poder económico y desprotección de los sectores más débiles; llegó un momento en que la alternativa fue el caos social, el enfrentamiento violento y la represión, o la incorporación de normas de justicia social, en términos de legislación protectora, seguridad social y redistribución. Quienes escogieron este último camino avanzaron hacia niveles de gran prosperidad y estabilidad. El dilema actual de la sociedad internacional es muy similar. El concepto del patrimonio económico de la humanidad equivale a optar por la incorporación de una justicia social internacional en el sistema económico, no como una herramienta inflexible sino como una pauta minima de acción mutuamente aceptable. Algunos podrán pensar que se trata de una utopia; quizás lo sea. Pero en todo caso es preferible experimentarla que esperar la alternativa cierta del colapso del actual sistema, con consecuencias imprevisibles respecto del orden mundial y de la preservación de la paz. 